\title{
Médiévales
}

Langues, Textes, Histoire

60 | printemps 2011

\section{La fitna}

\section{La fitna sicilienne : une fitna inachevée?}

The Sicilian Fitna : an Incomplete Fitna?

\section{Annliese Nef}

\section{(2) OpenEdition}

\section{Journals}

Édition électronique

URL : https://journals.openedition.org/medievales/6214

DOI : $10.4000 /$ medievales.6214

ISSN : 1777-5892

\section{Éditeur}

Presses universitaires de Vincennes

\section{Édition imprimée}

Date de publication : 30 juin 2011

Pagination : 103-115

ISBN : 978-2-84292-273-3

ISSN : 0751-2708

\section{Référence électronique}

Annliese Nef, «La fitna sicilienne : une fitna inachevée? », Médiévales [En ligne], 60 I printemps 2011, mis en ligne le 19 janvier 2012, consulté le 23 avril 2022. URL : http://journals.openedition.org/ medievales/6214; DOI : https://doi.org/10.4000/medievales.6214 
Médiévales 60, printemps 2011, p. 103-116

Annliese NEF

\section{LA F ITNA SICILIENNE: UNE FITNA INACHEVÉE?}

Le vocable fitna est très répandu dans les textes médiévaux islamiques, y compris si l'on s'en tient au champ du politique ou du politico-religieux, sans entrer dans la polysémie du terme ${ }^{1}$. Il connaît une gradation d'utilisation qui va de la fitna entre 'Ali et $\mathrm{Mu}^{\text {'awiya }}{ }^{2}$, voire des deuxième, troisième et quatrième fitna/s, distinguées par les historiens des premiers siècles de l'Islam et par les historiens contemporains à leur suite ${ }^{3}$, à des événements plus anecdotiques. On constate, en outre, que si les premières références affectent le centre de l'empire islamique, chaque région est susceptible d'avoir connu des épisodes qui sont considérés comme fondamentaux pour son évolution politique et sont taxés de fitna $^{4}$. Il reste certainement à mener une enquête systématique, mais une telle recherche suppose un travail d'équipe. Il s'agira donc ici de lever le voile sur ce qu'il en est de l'utilisation de ce terme pour la Sicile.

La Sicile est riche en dissensions internes et autres rébellions entre le début $\mathrm{du} \mathrm{x}^{\mathrm{e}}$ siècle, moment où les Fatimides prennent le pouvoir en Ifrîqiya et en Sicile, non sans opposition, et le milieu du $\mathrm{XI}^{\mathrm{e}}$ siècle, quand les divisions

1. Pour une définition du mot fitna, éminemment polysémique il est vrai, mais relativement clair par ses connotations quand il est utilisé dans le champ politique, même si chaque auteur en fait un usage contextuel, cf. à l'entrée «Fitna», dans l'Encyclopédie de l'Islam, $2^{\mathrm{e}}$ éd., Leyde, 19602009 et l'introduction au présent numéro.

2. On peut voir sur ce point, H. Duaїт, La Grande Discorde, Paris, 1989. Cet épisode est l'objet d'une bibliographie abondante que nous ne détaillons pas ici.

3. Cf. A. Borrut, Entre mémoire et pouvoir. L'espace syrien sous les derniers Omeyyades et les premiers Abbassides (v. 72-193/692-809), Leyde, 2010, qui rappelle la scansion de la première histoire omeyyade par un certain nombre d'affrontements politiques débouchant sur des manifestations militaires et qualifiés de fitna.

4. Cf. les exemples, dans ce numéro, qui traitent d'al-Andalus. 
internes sont avancées comme facteur décisif de la victoire des chrétiens, appelés dans l'île par une des multiples parties musulmanes qui s'opposent, un thème classique s'il en est. Autant d'événements qui renvoient à l'idée de division de la société, ou d'une partie de celle-ci, et pourraient être qualifiés de fitna par les sources arabo-musulmanes médiévales qui les évoquent, pourtant ils ne le sont que rarement dans le cas de la Sicile. Nonobstant cette histoire insulaire fitnogène, si l'on peut oser ce néologisme, le terme n'apparaît en effet que peu dans les sources qui retracent l'histoire de l'île. Nous essaierons donc d'avancer des raisons à même d'expliquer cette absence. Paradoxalement, l'absence de la fitna, entendue dans ses formes les plus graves et paradigmatiques, se lit quasiment comme un manque : toutes les grandes régions de l'Islam ont connu leurs fitna/s, en particulier les terres centrales de l'empire; puis, après l'apparition de califats concurrents ${ }^{5}$, chaque califat. Mais cette manifestation traumatique de la vie politique n'est pas réservée aux califats. La fitna semble quasiment constitutive de l'histoire et de la mise en place de la légitimation et de la défense de l'État dans le cadre de l'Islam médiéval.

Nous verrons d'abord que les occurrences du terme fitna sont relativement distinctes les unes des autres, ce qui fait douter d'un usage unanime du terme dans le cadre de l'histoire insulaire, comme ailleurs: le vocable fitna y retrouve en quelque sorte une partie de sa polysémie originelle. Une telle variété d'usages reflète en outre l'absence, à l'inverse de ce qui se passe ailleurs, de l'identification d'un moment qui serait LA fitna sicilienne; il s'agit donc plutôt de décliner les fitna/s siciliennes successives.

La source la plus importante pour l'histoire de la Sicile islamique et normande est la chronique universelle d'Ibn al-Athîr (1160-1233), complétée ou amendée par ses successeurs mais utilisée par tous les auteurs postérieurs, sans exception ${ }^{6}$. Cet auteur oriental présente en outre l'intérêt d'utiliser des sources occidentales qu'il ne cite pas ${ }^{7}$. Des trois historiens qui lui sont postérieurs et dont l'importance est notable, deux sont maghrébins, Ibn 'Idhârî (deuxième moitié du XIII ${ }^{\mathrm{e}}$ siècle - premières décennies du siècle suivant $)^{8}$ et le célèbre Ibn

5. Le califat fatimide apparu en 909 et celui des Omeyyades d'al-Andalus (929).

6. Nous ne détaillons ici que ce qui concerne les chroniques les plus importantes pour notre sujet, on trouvera des informations sur Ibn al-Khatîb plus loin.

7. Son Kâmil («Le parfait») est une chronique universelle qui court de la création du monde à l'année 1231 ; Kitâb al-kâmil, éd. C.J. ToRnBERG, Leyde, 1851-1876.

8. Son Bayân («Exposé») est un exposé analytique de l'histoire de l'Ifrîqiya entre 640 et 1205 , suivi d'une histoire d'al-Andalus, tandis que la troisième partie réunit des notices sur les Almoravides et les Almohades jusqu'à la fin de cette dynastie. Pour la première partie, l'édition de référence est celle de G.S. Colin et É. Lévi-Provençal, Histoire de l'Afrique du Nord et de l'Espagne musulmane intitulée Kitâb al-Bayân, Leyde, 1948-1951. 
Khaldûn (1332-1406) ${ }^{9}$, tandis que le plus tardif, al-Nuwayrî (1278 ou 12831332), est égyptien ${ }^{10}$.

Étant donnée l'influence d'Ibn al-Athîr sur les chroniqueurs postérieurs, on se penchera prioritairement sur les rares auteurs antérieurs, à la recherche d'informations provenant peut-être de sources distinctes. Deux textes retiennent ainsi l'attention. Al-Baladhûrî, d'une part, est un auteur oriental (m. 892), qui a rédigé un ouvrage de maghâzî considéré comme majeur ${ }^{11}$, mais qui traite peu de la Sicile, dont la conquête se déroule précisément au IX ${ }^{\mathrm{e}}$ siècle (entre 827 et 965). D'autre part, la Chronique de Cambridge, un texte anonyme daté au $\mathrm{XI}^{\mathrm{e}}$ siècle qui nous est parvenu dans deux versions indépendantes, l'une en grec et l'autre en arabe ${ }^{12}$, toutes deux débutant en 827 , la seconde s'interrompant avec l'année $964-965^{13}$.

Commençons par préciser que ces deux sources précoces, en particulier la Chronique de Cambridge qui est attribuée à un auteur sicilien, n'utilisent jamais le terme de fitna, tandis que la seconde emploie celui de harb («guerre») en cas de dissensions internes ou le verbe khâlafa 'alâ («s'opposer à»), en cas de rébellion contre le gouvernement.

\section{Quatre utilisations marquées par la diversité...}

On trouve dans les passages relatifs à la Sicile des sources plus tardives quatre occurrences du terme fitna dans des contextes d'utilisation et avec des significations très variés. Deux de ces épisodes se déroulent à la fin de la période aghlabide (800-909), un autre pendant la période de transition entre la fin de la domination de cette dynastie en Sicile et la mise en place de celle des Fatimides.

9. Ce savant maghrébin est l'auteur du Kitâb al-'Ibar («Livre des considérations édifiantes») qui se divise en deux parties: une histoire des Arabes (Mashreq) et celle des Berbères (dynasties du Maghreb): Ibn Khaldûn, Kitâb al-'Ibar, Le Caire, 1867-1868. Cet ouvrage a été analysé dans G. MartineZ-Gros, Ibn Khaldûn ou les Sept vies de l'Islam, Arles, 2006.

10. Al-Nuwayrî est l'auteur du Nihâyat al-'arab (Le Caire, 1955-1985), une œuvre encyclopédique qui vise à synthétiser les connaissances nécessaires aux secrétaires de l'administration de son époque, notamment en histoire.

11. Son Futûh al-Buldân («La conquête des pays») retrace les conquêtes islamiques depuis les origines; éd. M.J. De Goeje, Leyde, 1863-1866.

12. Pour la version arabe, cf. M. AMARI, Biblioteca arabo-sicula, rééd. revue par U. RizzITANo, Palerme, 1988 (Edizione nazionale delle opere di Michele Amari - Serie arabistica), vol.1, p. 190203 (cité désormais $B A S \S$ ar.), et pour les versions grecques Die byzantinischen Kleinchroniken, éd. P. SchreInER, vol.1, Einleitung und Texte, Vienne, 1975 (Corpus fontium historiae Byzantinae, 12, Series Vindobonensis), p. 326-340.

13. Ce texte mériterait un réexamen, annoncé, pour la version arabe conservée à Cambridge, par Jeremy Johns. 
Le dernier a lieu durant la période fatimido-kalbide, les Kalbides exerçant le pouvoir émiral dans l'île au nom des Fatimides à partir de 948.

\section{L'émirat d'Ibrâhîm II (875-902)}

Ce n'est peut-être pas un hasard si cet émir aghlabide dont la personnalité peut se prêter au développement de ce genre de débordements (il est connu pour des excès qui sont expliqués de manières variées) ${ }^{14}$, règne lors de la moitié de ces mentions.

La première ne se trouve que chez Ibn 'Idhârî, qui qualifie de fitna une opposition entre Berbères et Arabes en Sicile en 898-899 (285 h.) ${ }^{15}$. Ibrâhîm II appela alors au calme et à l'obéissance et fit punir quatre personnes dans des circonstances cruelles qui lui étaient coutumières. La mention est très elliptique et ne se retrouve que chez cet auteur, mais on peut noter que ce sont là les années où Ibrâhîm II mène en Ifrîqiya des campagnes féroces contre les Berbères ${ }^{16}$. Cet affrontement en est probablement un écho, même si cela n'est pas souligné par Ibn 'Idhârî. On pourrait également penser que la situation se rapproche de ce que l'on connaît pour al-Andalus, notamment au XI siècle. Mais le contexte y est très différent de celui de la Sicile dans la mesure où, dans la péninsule ibérique, le thème est récurrent, tout comme les affrontements dans lesquels cette dimension est mise en avant, quelle que soit la réalité de leur connotation ethnique. Or, pour ce qui concerne la Sicile, les Berbères sont quasiment absents des sources, alors même qu'il est probable qu'ils composaient une partie des Ifrîqiyens installés en Sicile ${ }^{17}$.

À propos d'événements qui se sont déroulés un à deux ans plus tard, dans d'autres circonstances, le terme est employé cette fois par Ibn Khaldûn ${ }^{18}$. En 900 (287 h.), le fils d'Ibrâhîm II, Abû 'Abd Allâh al-'Abbâs, est envoyé en Sicile par son père pour y reprendre les choses en main. En effet, une fitna vient d'y opposer les habitants d'Agrigente à ceux de Palerme, chacune des deux villes ayant fait

14. A. NEF, «Violence and the Prince : the Case of the Aghlabid Amîr Ibrâhîm II (261-289/875902)», dans M. Fierro et C. Lange éd., Public Violence in Islamic Societies. Power, Discipline, and the Construction of the Public Sphere, 7th-19th Centuries CE, Edinburgh, 2009, p. 217-237.

15. Cf. IBN 'IDHÂR̂̂, Bayân, BAS it., II, p. 19 et $B A S$ ar., II, p. 417: «Kânat fitna bi-Siqilliya bayn 'Araba-hâ wa-Barbara-hâ.»

16. Cf. A. NEF, «Violence and the Prince.... :», p. 221 et V.PrÉvost, L'Aventure ibadite dans le Sud tunisien (VIII'-XIII siècle). Effervescence d'une région méconnue, Helsinki, 2008, p. 97-99, à propos de l'affrontement de Mānū, en 896, et des années qui suivent.

17. Cf. L. Chiarelli, «The Ibadi presence in Muslim Sicily», Bulletin of the Royal Institute for Inter-Faith Studies, 7/1 (2005).

18. IBn Khaldûn, Kitâb al-'Ibâr, Biblioteca arabo-sicula, rééd. Catane, 1982 (désormais abrégé $B A S$ it.), II, p. 185 et $B A S$ ar., II, p. 526. Le même épisode est qualifié cette fois de malhama («bataille féroce», «massacre») par Ibn 'Idhârî, Bayân, BAS it., II, p. 20 et BAS ar., II, p. 417. 
appel au fils de l'émir contre l'autre. Son arrivée et sa volonté de rétablir l'ordre provoquent alors l'union des ennemis d'hier contre lui. Néanmoins, les causes de cette fitna sont pour le moins peu claires: s'agissait-il là d'un prolongement des troubles antérieurs ${ }^{19}$ - mais, dans ce cas, pourquoi Ibn 'Idhârî ne qualifiet-il pas ce nouvel épisode de fitna ? -, de l'affrontement entre les élites d'une ville importante de Sicile et celles de sa capitale (pour des raisons qui peuvent être très diverses) ?

Ces exemples précoces mettent donc l'accent sur la division interne que suppose la fitna mais sans que l'usage du terme soit systématique, ni le contexte de ces deux occurrences identique! Une question se pose toutefois, à laquelle seule une enquête systématique sur l'emploi du mot fitna dans le cadre de l'histoire du Maghreb médiéval permettrait de répondre: la grille de lecture qui consiste à superposer tensions entre groupes définis comme berbères et groupes définis comme arabes et fitna est-elle une grille de lecture récurrente, au-delà de sa pertinence, dans les sources maghrébines? La réponse à cette question autoriserait à établir si la Sicile, pour l'histoire de laquelle cette explication est peu avancée par les auteurs médiévaux, se voit appliquer dans ce passage une interprétation élaborée dans un cadre maghrébin.

\section{Les Fatimides}

Deux épisodes sont à distinguer: le premier se déroule dans les années 910 et coöncide avec la révolte d'Ibn Qurhub contre les Fatimides, dont le califat vient d'être proclamé en Ifrîqiya. Ibn Qurhub choisit alors de se rallier aux Abbassides avec ses partisans. Ce geste est suivi par une fitna entre soutiens et opposants du rebelle. Deuxième événement: dans les années 930, à la suite d'une révolte qui a secoué l'île, une reprise en main par les Fatimides a lieu. Là aussi, le terme fitna est utilisé.

19. Nombre d'historiens ont considéré que Palerme était la représentante des «intérêts arabes» en Sicile et Agrigente celle des «intérêts berbères ». Les sources suggèrent plutôt une concentration de certains groupes constitutifs du jund ifrîqiyen à Palerme (les Banû Tabarî ou les Banû Aghlab par exemple), dont une partie revendique une ascendance arabe et une opposition entre deux capitales régionales, les deux éléments n'étant pas nécessairement lisibles en termes d'affrontement entre Arabes et Berbères. Cf. A. Nef, «Les armées arabo-musulmanes en Sicile et en Italie du Sud (IX ${ }^{\mathrm{e}}-\mathrm{X}^{\mathrm{e}}$ siècle) : composition des troupes et silences des sources », dans D. BARTHÉLÉMY et J.-C. CHEYNET éd., Guerre et Société au Moyen Âge, Byzance-Occident (VIII'-XIII siècle), Paris, 2010, p. 85-100. 


\section{Ibn Qurhub}

En 909, al-Mahdî, nouveau calife fatimide, qui vient de mettre fin au pouvoir des Aghlabides en Ifrîqiya, entend bien en faire autant en Sicile ${ }^{20}$. Il désigne donc en 910-911 al-Hasan b. Ahmad b. Ibn Abî Khinzîr, qui était alors gouverneur de Kairouan, comme émir de Sicile. Toutefois, rapidement, ce dernier et son frère 'Alî, qu'il a désigné à la tête d'Agrigente, se voient reprocher leur brutalité par la population. Se rendant aux arguments de cette dernière, le calife envoie un certain 'Alî b. 'Umar al-Balawî dans l'île pour remplacer l'émir palermitain. Le nouveau gouverneur, critiqué cette fois pour son âge avancé et sa faiblesse, est rapidement renversé par la population insulaire qui porte à sa tête Ahmad b. Qurhub, en raison de ses qualités, alors même qu'il n'aurait pas vraiment recherché cette lourde charge ${ }^{21}$. Une fois en fonction, Ibn Qurhub développe une double politique d'affrontement: avec les chrétiens d'abord, puis, à partir de 912, avec les Fatimides.

En 912-913, Ibn Qurhub décide ainsi de s'attaquer à Taormine qui échappe encore au contrôle islamique. Toutefois, la ville résiste et le siège dure trois mois, provoquant une sédition au sein de l'armée, lasse de cette opération infructueuse. C'est à la suite de cet échec qu'Ibn Qurhub appelle à prêter obédience à al-Muqtadir, le calife abbasside, afin de rétablir sa légitimité chancelante. Il cesse alors de prononcer la khutba au nom du calife fatimide et reçoit des drapeaux et des vêtements envoyés par le calife abbasside, lesquels symbolisent son investiture par le pouvoir de Bagdad. Soulignant encore davantage le rejet sicilien des nouveaux maîtres de l'Ifrîqiya, il attaque une partie de la flotte fatimide en 914 le long des côtes de l'Ifrîqiya, à la hauteur de Lamta. Les prisonniers se comptent par centaines, parmi lesquels Ahmad b. Abî Khinzîr, déjà évoqué, qui est mis à mort ${ }^{22}$.

Ibn Qurhub continue de se battre sur deux fronts - l'Ifrîqiya, avec moins de succès désormais, et la Calabre byzantine -, mais la population le craint toujours plus et les Agrigentins finissent par se rebeller contre lui. Les Siciliens écrivent alors au calife fatimide pour se soumettre à lui et réclamer son aide: ce sont les ahl al-bilâd («élites des localités ») de la Sicile qui prennent ainsi à témoin le mahdî. Ils lui expliquent que leur démarche est motivée par leur « haine

20. Sur la transition entre les deux dynasties, cf. M. TALBI, L'Émirat aghlabide. Histoire politique, Paris, 1966, chap. 7 et M. BRETT, The Rise of the Fatimids. The World of the Mediterranean and the Middle East in the Fourth Century of the Hijra, Tenth Century CE, Leyde-Cologne-Boston, 2001, chapitre 4.

21. IBN AL-AtнîR, Kitâb al-Kâmil, BAS it., I, p. 408-409 et BAS ar., I, p. 296. Cf. également H. Halm, The Empire of the Mahdi. The Rise of the Fatimids, trad. de Das Reich des Mahdi. Der Aufstieg der Fatimiden, Munich, 1991, Leyde-New York-Cologne, 1996, p. 177-178.

22. IBn AL-Atнîr, Kitâb al-Kâmil, BAS it., I, p. 409 et BAS ar., I, p. 296. 
de la fitna ${ }^{23}{ }$, au sens de division interne, rébellion, si l'on en croit Ibn al-Athîr. Ils livrent alors Ibn Qurhub et ses proches aux Fatimides, lesquels les mettent à mort sur la tombe de Ibn Abî Khinzîr en 916 (303 h.) ${ }^{24}$.

L'utilisation du terme à propos des mêmes événements est légèrement décalée chez Ibn 'Idhârî: en 912-913 (300h.), il précise que l'île s'oppose (khâlifat) aux Fatimides ${ }^{25}$. En 915-916 (303 h.), lorsque les premiers succès d'Ibn Qurhub contre les Fatimides sont suivis par des revers qui entraînent le soulèvement d'une partie des Siciliens contre son autorité, ce dernier tente en vain de les convaincre de revenir au calme. C'est alors qu'Ibn 'Idhârî utilise le terme fitna pour décrire l'opposition entre partisans et opposants d'Ibn Qurhub qui déchire l'île, au point que l'auteur utilise le mot tâ'ifa («parti») ${ }^{26}$ pour désigner chacune des factions. Il est probable que cette opposition était sousentendue chez Ibn al-Athîr.

Si les contextes d'utilisation sont bien ici toujours ceux d'une division des Siciliens, le choix du mot fitna semble justifié par l'extension et la violence du conflit, mais aussi par sa dimension fratricide. Le terme tâ'ifa introduit, en outre, un élément qui semble nouveau: celui d'une lutte explicitement menée en vue de s'emparer du pouvoir.

Le deuxième épisode fatimide, qui se déroule en 936-937, a beaucoup plus directement à voir avec un positionnement par rapport aux Fatimides et est moins médiatisé par un troisième terme/individu que cela n'était le cas sous l'émirat d'Ibn Qurhub.

\section{L'année 936-937 et l'intervention de Khalîl}

Pour décrire les événements de l'année 936-937 (325h.), Ibn al-Athîr intitule son paragraphe: Dhikr al-fitna bayn ahl Siqilliyya wa-umarâ'-hum («Récit de la fitna entre la population de Sicile et ses émirs») ${ }^{27}$. Il est le seul à le faire. Reprenons donc son récit de cette fitna afin de comprendre pourquoi. Tout d'abord, les Agrigentins se rebellent contre Sâlim b. Râshid, l'émir de Sicile mis en place par al-Qâ'im, parce qu'il s'était mal comporté à leur égard. Ils chassent le percepteur ('âmil) désigné par l'émir et une armée est envoyée contre la ville. L'affrontement se solde par la défaite de l'armée émirale; les Agrigentins poussent alors leur avantage vers Palerme et sont vaincus. Les

23. L'expression exacte est: karahû al-fitna, Ibid., BAS it., I, p. 410 et BAS ar., I, p. 297.

24. Ibid.

25. IBN 'IDHÂRî, Bayân, BAS it., II, p. 23 et $B A S$ ar., I, p. 418-419.

26. IBN 'IDHÂRî, Bayân, BAS it., II, p. 24 et BAS ar., I, p. 419. Le mot tâ'ifa est également polysémique. Dans le sens de faction ou de parti politique, on le retrouve, presque exemplairement, pour l'histoire d'al-Andalus.

27. Iвn AL-Aтнîr, Kitâb al-Kâmil, BAS it., I, p. 413 et BAS ar., I, p. 299. 
Palermitains se rebellent à leur tour et sont matés, mais l'émir appelle le calife à la rescousse car ces derniers «sont sortis de l'obéissance qu'ils lui devaient et se sont rebellés contre lui» (kharajû 'an tâ'ati-hi wa-khâlafû 'alay-hi). Toutefois, et sans entrer dans les détails, les choses ne s'arrêtent pas là : le calife envoie alors Khalîl b. Ishâq ${ }^{28}$ sur place ${ }^{29}$. Ce dernier écoute les plaintes des Palermitains, mais construit surtout au sud-est de la ville, à proximité immédiate de cette dernière et le long de la côte, une cité administrative fortifiée, la Khâlisa, qui permet le contrôle de la population de Palerme, et probablement aussi un départ précipité par mer si nécessaire. Les Agrigentins, quant à eux, poursuivent leur mouvement d'opposition et sont assiégés durant huit mois.

En 938-939, la rébellion s'étend à l'ensemble des points fortifiés de Sicile et à Mazara à l'instigation des Agrigentins. Dans ce contexte, le verbe utilisé est celui de khâlafa, comme plus haut ${ }^{30}$. Si les forteresses sont défaites, la population d'Agrigente persiste et fait appel à Constantinople qui lui envoie vivres et hommes. Lorsque la ville tombe, tous ceux qui n'ont pas gagné le pays des Rûm sont emmenés et les représentants des élites agrigentines demeurés sur place sont noyés en mer.

La description de cet épisode par Ibn al-Athîr est donc fidèle au titre de la section: il s'agit plus d'une rébellion que d'une division interne, ce qui rétrospectivement explique son premier usage du terme fitna mentionné plus haut à propos des années 915-916. Les élites siciliennes désapprouvaient alors la rébellion des Agrigentins. En même temps, le second usage du terme est curieux car la construction avec bayn («entre») fait attendre l'opposition de deux camps: s'agit-il des sujets et des représentants de l'autorité ? Probablement le terme est-il plutôt utilisé, comme dans le premier cas, pour sa connotation classique, en référence à des déchirures profondes de la société islamique. En effet, la violence et la durée de la rébellion menée par Agrigente, sa trahison au profit de Constantinople et son châtiment dépourvu de toute indulgence semblent justifier cette utilisation. Dans le cas de la première occurrence due à Ibn al-Athîr, il s'agissait d'exprimer une détestation profonde du désordre défavorable à l'unité de l'umma .

On voit donc que les occurrences de ce terme pour renvoyer à un contexte sicilien sont aussi rares que variées: aucun épisode n'est unanimement évoqué par les auteurs médiévaux comme LA fitna sicilienne, y compris en précisant comme

28. Il s'agit d'un des plus grands chefs militaires de l'armée fatimide: il était originellement à la tête du jund de Kairouan et aida les Fatimides à conquérir le pouvoir, puis à étendre leur emprise au Maghreb. Cf. M. BRETt, The Rise of the Fatimids..., p. 175 et 270.Il s'agit donc d'un homme de confiance.

29. Iвn AL-Athîr, Kitâb al-Kâmil, BAS it., I, p. 413-414 et BAS ar., I, p. 299.

30. IвN AL-AтнîR, Kitâb al-Kâmil, BAS it., I, p. 414 et BAS ar., I, p. 300. 
on le faisait dans les cas où des événements de ce type s'étaient succédés, par un qualificatif, une date ou une numérotation. Sont évoqués des affrontements entre groupes, entre villes, entre partis politiques et une rébellion. En outre, les mêmes événements ne sont pas décrits par le même vocable chez les différents auteurs et si Ibn Khaldûn et Ibn 'Idhârî font un usage relativement classique du mot fitna pour renvoyer à l'opposition de deux camps, Ibn al-Athîr semble l'utiliser plus pour ce qu'il évoque que pour ce qu'il signifie.

\section{... et un absent surprenant !}

Le reste du temps, le vocable fitna n'apparaît pas, y compris à la fin de la période islamique, lorsque se mettent en place sur les cendres de l'émirat kalbide de Sicile, dans les années 1040, des entités autonomes comparables à celles des mulûk al-tawâ'if («rois de Taïfas») selon la terminologie andalousienne.

En s'en tenant à Ibn al-Athîr, qui est la source fondamentale pour l'histoire de la Sicile islamique, à partir de 947-948 (date de l'avènement en Sicile du premier émir kalbide désigné par les Fatimides, lequel va donner naissance à une dynastie plus ou moins autonome), le terme disparaît. Et pourtant une série d'épisodes pourraient se prêter à cette qualification ; passons-les rapidement en revue.

En 969-970 (359 h.), un mawlâ ${ }^{31}$ des Kalbides, Ya'îsh, est envoyé dans l'île; il rassemble pour une raison peu claire les qabâ'il (tribus, clans) dans l'arsenal et ceux-ci affrontent les mawâlî des Kutâma, célèbre tribu berbère. On peut avancer deux hypothèses: soit le premier groupe désigne les représentants des lignages qui se proclamaient arabes et avaient été proches des Aghlabides, et il s'oppose à des Berbères qui soutenaient les Fatimides ${ }^{32}$, soit cet épisode reflète des tensions entre différents groupes connus pour leur soutien aux Fatimides. Dans ce cas, les premiers, de toute évidence proches des Kalbides, doivent représenter le versant qualifié d' «oriental» dans les sources, tandis que les seconds sont clairement du côté des «Occidentaux ${ }^{33}$. On sait que ces groupes pouvaient agir en tant que factions politiques autour du calife fatimide et il est

31. Le sens de ce terme est complexe et varie au cours de l'histoire de l'Islam, mais il renvoie à la notion de clientèle.

32. Sur ces deux points, cf. note 19 et A. NEF, «Les armées arabo-musulmanes...».

33. L'origine des Fatimides est orientale (Salamya a été le premier centre de prédication des Ismâ'iliens) et les sources qui rapportent l'arrivée des premiers représentants des Fatimides dans la région les qualifient toujours d'«Orientaux» (Mashâriqa). Les Occidentaux dans les sources se rapportant aux Fatimides sont, quant à eux, leurs soutiens d'origine maghrébine, une fois que les Fatimides sont passés en Égypte, soit après 969 : ils sont, par exemple, présents, en tant que tels, dans les processions califales au Caire: cf. P. SAnders, Ritual, Politics, and the City in Fatimid Cairo, Albany, 1994, p. 124. 
probable qu'il s'agit là d'un écho sicilien de semblables tensions. L'origine et les causes des événements siciliens ne sont pas claires, mais le terme employé est celui de sharr ( «méfait», «injustice» entre autres). Lorsque l'agitation, qui porte à des déprédations, notamment contre les dhimmî-s, s'étend à Syracuse, le vocable utilisé demeure le même. Yâ'ish est remplacé par un Kalbide et l'agitation prend fin ${ }^{34}$.

La dernière partie du récit d'Ibn al-Athîr vise à expliquer la conquête de l'île par les Normands en 1092-1093 et sa perte par l'Islam. Or, dans ce but, le chroniqueur remonte explicitement en arrière dans le temps, jusqu'à l'année 998 ! À cette date, le Kalbide Yusûf est émir. Frappé par une hémiplégie, il est remplacé par son fils Ja 'far qui règne sans problème jusqu'en 1014, date à laquelle son frère, 'Alî, se rebelle contre lui (khâlafa 'alay-hi), soutenu par des esclaves et des Berbères. Ja 'far lui envoie alors le jund qui le défait et 'Alî, fait prisonnier, est mis à mort. L'émir en place aurait alors chassé les Berbères de l'île et fait mettre à mort les esclaves, réservant le jund aux seuls Siciliens. La diminution des effectifs de ce dernier et son affaiblissement consécutif seraient la cause d'une dégradation du gouvernement qui, à son tour, aurait entraîné une révolte. Pour y mettre fin, Yûsuf lui-même, âgé et diminué, apparaît à la vue des rebelles et leur annonce un changement d'émir, son autre fils Ahmad al-Akhal prenant le pouvoir ${ }^{35}$. Cette série d'événements, qui semble propice à l'utilisation du terme fitna, ne le voit pourtant pas utilisé une seule fois.

D'autant que les choses ne s'arrêtent pas là: al-Akhal propose aux Siciliens de chasser les Ifrîqiyens, mais se heurte à un refus de leur part. Il fait alors la proposition inverse aux Ifrîqiyens qui acceptent. Du coup, les Siciliens auraient été taxés du kharâj, impôt foncier en théorie réservé aux non-musulmans, alors même qu'ils étaient musulmans ${ }^{36}$. Ils en appellent alors au calife fatimide et menacent de faire allégeance aux Rûm-s. Ce dernier envoie une armée qui assiège al-Akhal et le tue, entraînant un émoi dans la population (thumma ikhtilafa ahl Siqilliyy ${ }^{37}$ ).

Dans les deux cas, les camps qui s'affrontent sont présentés comme des groupes potentiellement antagonistes mais, là encore, la fitna ne fait pas son apparition, peut-être parce que le tout est présenté comme une manipulation due

34. Pour tout ce passage: IBn AL-AthîR, Kitâb al-Kâmil, BAS it., I, p. 429-430 et BAS ar., I, p. 308-309.

35. IBN AL-AthîR, Kitâb al-Kâmil, BAS it., I, p. 442-444 et BAS ar., I, p. 316-317.

36. IвN AL-Aтнîr, Kitâb al-Kâmil, BAS it., I, p. 444 et BAS ar., I, p. 316.

37. IвN AL-AтнîR, Kitâb al-Kâmil, BAS it., I, p. 445 et BAS ar., I, p. 317. 
à des émirs et non comme un mouvement spontané. Aucun des auteurs passés en revue ne fait exception ${ }^{38}$.

Enfin, le plus étonnant est sans doute que la division de l'île en cinq entités autonomes en guerre permanente, à partir de 1040 environ - une division qui débouche sur l'appel aux Normands de Calabre - n'est pas non plus qualifiée de fitna par les auteurs retenus.

\section{Une fitna sicilienne inachevée?}

La Sicile offre donc un visage paradoxal: dans les sources disponibles, revient à plusieurs reprises l'idée que les Siciliens sont particulièrement difficiles à gouverner, qu'ils se rebellent fréquemment et qu'ils sont divisés, mais le terme de fitna n'est que peu employé, alors même qu'il peut recouvrir tous ces sens. La question est donc d'expliquer cette absence. On peut envisager plusieurs pistes, tout en insistant sur le fait que l'on ne pourra véritablement répondre à cette question qu'avec davantage de données sur l'utilisation du vocable chez chacun des auteurs passés en revue et de manière plus générale dans les sources de l'histoire du monde islamique médiéval.

1) Il est probable que ce trait découle en partie de la volonté qui anime Ibn al-Athîr de présenter la Sicile comme caractérisée par l'unité du camp musulman qui s'oppose aux chrétiens désunis ${ }^{39}$, d'autant que la conquête de l'île s'achève dans les années 960. Jusqu'à cette date, il fait de cette opposition musulmans/chrétiens la grille d'interprétation de l'histoire sicilienne et, s'il montre l'affaiblissement interne de la Sicile à partir de la fin du $x^{e}$ siècle, il tend constamment à atténuer les divisions intra-musulmanes. Or, il sert de source à quasiment tous les auteurs postérieurs.

2) Cela va-t-il également de pair avec une explication de ces troubles récurrents qui est donnée dans un texte de cosmographie découvert récemment et qui était peut-être partagée, même si elle n'est pas explicitement avancée sous cette forme, par d'autres auteurs? Anonyme, datant de 1050 environ et agrémenté d'une carte avec didascalies ${ }^{40}$, le Kitâb Garâ'ib al-funûn a été rédigé par un auteur favorable aux Fatimides. Il s'inspire en partie d'Ibn Hawqal pour sa description de la Sicile, tout y apportant des modifications et des ajouts, parmi lesquels figure ce passage final:

38. Al-Nuwayrî utilise des termes venant de la racine $k h . l . f . ;$ Ibn Khaldûn également qui décline aussi la racine th.w.r.

39. Sur ce point, cf. A. NEF, «Les armées arabo-musulmanes...».

40. Cf. J. Johns, «Una nuova fonte per la geografia e la storia della Sicilia nell'xi secolo: il Kitâb Garâ'ib al-funûn wa-mulah al- ‘uyûn», Mélanges de l'École française de Rome, 116/1 (2004), p. 409-449. 
Les astrologues affirment que quand la maison du Lion monte obliquement, elle exerce, en dépit de sa réputation bénéfique, une influence maléfique, si bien que tout pays sous cette influence est difficile à gouverner pour le souverain $(s a$ c $b a l$ tâ 'a 'alâ al-sultân wa-huwa yatawilâ). Samarcande, Ardabîl, la Mecque, Damas et la Sicile: ces pays ne sont pas en accord (salaha li-) avec leurs gouvernants, ni leurs gouvernants avec eux ${ }^{41}$.

Cette idée était-elle devenue un lieu commun qui ne méritait même pas d'être rappelé explicitement par les auteurs que nous avons passés en revue? La récurrence de références à une sorte d' «esprit sicilien » pourrait le faire penser ${ }^{42}$, même si cette explication a pu être oubliée, ou ne pas être reprise, ou n'être développée que par certains.

3) Mais on peut avancer, plus largement, que cette sorte de manque reflète l'inachèvement du processus de la fitna en Sicile: la rébellion, le désordre politique, l'affrontement entre factions va rarement jusqu'à l'apparition de nouvelles entités politiques plus ou moins stables et rivales (celles de la fin de la période ne durent guère plus d'une décennie et, même si elles sont mal connues, ne semblent guère stables).

Au fond n'est-ce pas le critère de ce qui définit la «véritable» fitna en Islam médiéval? Elle construirait - ou tenterait de construire - une légitimité autre que celle en place, le revers de la légitimité étant le trou noir de la fitna en Islam. On a l'impression que le déroulement de fitna/s importantes est un des critères de reconnaissance de l'appartenance pleine et entière d'une région au dâr al-islâm, tandis que la question de la légitimité en Sicile se pose toujours par rapport à un référent extérieur, si l'on en croit les sources dont on dispose. Peut-être cela découle-t-il aussi de l'idée que la Sicile est conquise tardivement par rapport aux premières fitna/s de l'Islam, que sa conquête est lente et qu'elle constitue une sorte d'horizon du jihâd, plutôt qu'un espace où se construit une légitimité politique assise. Contrairement à l'ensemble des autres régions du dâr al-islâm, jamais, sauf durant la dernière décennie de la domination islamique, la Sicile n'a été gouvernée par une entité complètement autonome.

Il conviendrait donc d'élargir l'enquête à l'ensemble des terres de l'Islam afin de confirmer ou d'infirmer cette hypothèse.

Annliese Nef - Université Paris-Sorbonne - UMR 8167, Institut universitaire de France, UFR d'histoire, 1 rue Victor Cousin, F-75005 Paris

41. Ibid. p. 432, pour la version arabe et p. 437, pour la version italienne.

42. Ainsi Ibn HaQwal lui-même évoque les fitna-s des occupants des ribât-s siciliens contre lesquels il n'a pas de mots assez durs: cf. IBn HaQwal, Surât al-ard, Beyrouth, 1964, p. 116 et I. HaQwal, Configuration de la terre, trad. J.H. Kramers et G. Wiet, Paris, 1964, p. 120: «Les bâtiments se sont écroulés et leurs pensionnaires ont péri à la suite des troubles (al-fitan, pl. de fitna) et des rébellions qu'ils ont causés eux-mêmes pour se soustraire à l'autorité du prince.» 


\section{La fitna sicilienne : une fitna inachevée?}

La Sicile islamique ( $\left(\mathrm{IX}^{\mathrm{e}}-\mathrm{XI}^{\mathrm{e}}\right.$ siècle) semble peu fertile en événements qualifiés de fitna dans les sources. Seules quatre occurrences du terme apparaissent au cours d'un peu plus de deux siècles de domination musulmane de l'île. $\mathrm{Ou}$ plus exactement, des épisodes semblables à d'autres qui seraient appelés de la sorte ailleurs ne le sont pas en Sicile et la qualification d'un événement en fitna n'est jamais unanime chez les auteurs consultés. Les raisons de cette absence sont difficiles à établir de manière certaine, mais cette dernière semble refléter l'intégration inachevée de la Sicile à l'ensemble politique islamique aux yeux des auteurs médiévaux.

Sicile islamique - vocabulaire politique - fitna - Fatimides - Aghlabides.

\section{The Sicilian Fitna: an Incomplete Fitna?}

Islamic Sicily (ninth-eleventh century) did not give birth to many events qualified of fitna in the sources. Only four occurrences of the word have been found during the more than two centuries of muslim domination over the island. Or, more exactly, events similar to others which are called fitna/s elsewhere are not qualified as such in Sicily. Moreover, the designation of an event as a Sicilian fitna is never unanimous among the authors. The reasons of this absence are difficult to establish with certainty, but it could reflect the incomplete political integration of Sicily into the Islamic world in the eyes of the medieval authors.

Islamic Sicily - political vocabulary - fitna - Fatimids - Aghlabids. 
